\title{
Shared genetics of asthma and mental health disorders: a large-scale genome- wide cross-trait analysis
}

\author{
Zhaozhong Zhu ${ }^{1,2,3}$, Xi Zhu ${ }^{4}$, Cong-Lin Liu ${ }^{5}$, Huwenbo Shi ${ }^{1}$, Sipeng Shen ${ }^{3}$, \\ Yunqi Yang ${ }^{1}$, Kohei Hasegawa ${ }^{2}$, Carlos A. Camargo Jr (1) ${ }^{1,2}$ and Liming Liang ${ }^{1,6}$ \\ Affiliations: ${ }^{1}$ Program in Genetic Epidemiology and Statistical Genetics, Dept of Epidemiology, Harvard T.H. \\ Chan School of Public Health, Boston, MA, USA. ${ }^{2}$ Dept of Emergency Medicine, Massachusetts General \\ Hospital, Harvard Medical School, Boston, MA, USA. ${ }^{3}$ Dept of Environmental Health, Harvard T.H. Chan School \\ of Public Health, Boston, MA, USA. ${ }^{4}$ Dept of Critical Care Medicine, Peking University Third Hospital, Beijing, \\ China. ${ }^{5}$ Dept of Medicine, Brigham and Women's Hospital and Harvard Medical School, Boston, MA, USA. \\ ${ }^{6}$ Dept of Biostatistics, Harvard T.H. Chan School of Public Health, Boston, MA, USA.
}

Correspondence: Zhaozhong Zhu, Depts of Epidemiology and Environmental Health, Harvard T.H. Chan School of Public Health and Dept of Emergency Medicine, Massachusetts General Hospital, Harvard Medical School, 665 Huntington Avenue, Building 1, 1406C, Boston, MA 02115, USA. E-mail: zhz586amail.harvard.edu

@ERSpublications

This study discovered shared genetic components between asthma and ADHD, anxiety and depression. The shared pathways and potential causal effects from mental disorders to asthma highlight a healthcare focus among patients with these disorders. http://bit.ly/2MsALoa

Cite this article as: Zhu Z, Zhu X, Liu C-L, et al. Shared genetics of asthma and mental health disorders: a large-scale genome-wide cross-trait analysis. Eur Respir J 2019; 54: 1901507 [https://doi.org/10.1183/ 13993003.01507-2019].

ABSTRACT Epidemiological studies demonstrate an association between asthma and mental health disorders, although little is known about the shared genetics and causality of this association. Thus, we aimed to investigate shared genetics and the causal link between asthma and mental health disorders.

We conducted a large-scale genome-wide cross-trait association study to investigate genetic overlap between asthma from the UK Biobank and eight mental health disorders from the Psychiatric Genomics Consortium: attention deficit hyperactivity disorder (ADHD), anxiety disorder (ANX), autism spectrum disorder, bipolar disorder, eating disorder, major depressive disorder (MDD), post-traumatic stress disorder and schizophrenia (sample size 9537-394283).

In the single-trait genome-wide association analysis, we replicated 130 previously reported loci and discovered 31 novel independent loci that are associated with asthma. We identified that ADHD, ANX and MDD have a strong genetic correlation with asthma at the genome-wide level. Cross-trait meta-analysis identified seven loci jointly associated with asthma and ADHD, one locus with asthma and ANX, and 10 loci with asthma and MDD. Functional analysis revealed that the identified variants regulated gene expression in major tissues belonging to the exocrine/endocrine, digestive, respiratory and haemic/immune systems. Mendelian randomisation analyses suggested that ADHD and MDD (including 6.7\% sample overlap with asthma) might increase the risk of asthma.

This large-scale genome-wide cross-trait analysis identified shared genetics and potential causal links between asthma and three mental health disorders (ADHD, ANX and MDD). Such shared genetics implicate potential new biological functions that are in common among them.

This article has supplementary material available from erj.ersjournals.com

Data availability: UK Biobank GWAS summary statistics will be available at the NHGRI-EBI GWAS Catalog website (www.ebi.ac.uk/gwas) or contact Zhaozhong Zhu for inquires about the GWAS data.

Received: 12 Apr 2019 | Accepted after revision: 26 Sept 2019

Copyright OERS 2019 


\section{Introduction}

Asthma is one of the most common chronic diseases, resulting in a substantial burden of disease worldwide. Accumulating studies have shown significant association between asthma and mental health disorders, such as anxiety, depression and attention deficit hyperactivity disorder (ADHD) [1]. Although consensus has emerged from the clinical, psychiatric and biological literature that psychosocial factors affect asthma pathobiology in both children and adults [2, 3], their role in the pathobiology, morbidity and symptomatology of asthma remains controversial [4]. For example, a recent large-scale systematic review and meta-analysis by CORTESE et al. [3] supports a significant phenotypic association between asthma and ADHD in both children and adults after controlling for possible confounders. LEHTO et al. [5] also recently found shared genetic influences between asthma and depression and high neuroticism, but not anxiety, based on genome-wide genetic correlation and polygenic risk scores. However, the shared genetics between asthma and ADHD, potential genetic causal effect and direction, specific shared genetic variants, and underlying mechanisms are still unknown for these traits.

We and colleagues have recently identified shared genetic architecture among respiratory, immune, cardio-metabolic and neurological/mental health disorders [6-9], indicating the potential pleiotropic effect. Asthma and mental health disorders are both highly heritable traits $[10,11]$. Parallel epidemic trends in asthma and mental health disorders worldwide suggested shared genetic and environmental components for both these conditions [1]. However, there is limited knowledge about the shared genetic components between asthma and mental health disorders. Furthermore, asthma is a highly heterogeneous disease. Recent studies showed the genetic background of childhood- and adult-onset asthma can be partly distinct $[12,13]$. Therefore, it is unclear if the shared genetics between asthma and mental health disorders can differ in these asthma subtypes.

In the current study, we conducted a large-scale genome-wide association study (GWAS) for cross-trait analysis between asthma from the UK Biobank and eight mental health disorders from the Psychiatric Genomics Consortium (PGC): ADHD, anxiety disorder (ANX), autism spectrum disorder (ASD), bipolar disorder (BIP), eating disorder (ED), major depressive disorder (MDD), post-traumatic stress disorder (PTSD) and schizophrenia (SCZ). Specifically, we investigated the genome-wide genetic correlation between asthma and these mental health disorders, and used cross-trait meta-analysis to identify shared individual genetic variants between them [14]. We carried out further GWAS functional analysis to delineate the biological impact of such shared genetics. Finally, we investigated the shared genetics between asthma and mental health disorders by childhood- and adult-onset asthma subtypes.

\section{Methods}

Study population, design, data summary and quality control

The overall study design is shown in figure 1. In this current study, we included two major data sources: the UK Biobank and the PGC.

\section{UK Biobank data}

The details of the UK Biobank cohort are described elsewhere [15] and in the supplementary material. All participants provided informed consent to the UK Biobank. We performed a stringent sample quality control procedure. We restricted the sample set to a European population using genetic ancestry based on principal components analysis of the genotypes (data field 22006). We excluded individuals with chronic obstructive pulmonary disease, emphysema or chronic bronchitis (self-reports or International Statistical Classification of Diseases and Related Health Problems, 10th Revision codes) from asthmatic cases and controls. Asthma was treated as the primary phenotype of interest. Three asthma subtypes were treated as secondary phenotypes and were defined in this study as: childhood-onset asthma (age of onset $\leqslant 12$ years), adult-onset asthma (age of onset $\geqslant 26$ years) and young adult-onset asthma (age of onset $>12-25$ years). Young adult-onset asthma was not included in the genetic analysis due to its higher heterogeneity $[12,13]$. Thus, 46802 asthma cases, 9676 childhood-onset asthma cases, 22296 adult-onset asthma cases and 347481 shared controls with high-quality genotyping and complete phenotype/covariate data were included for GWAS analysis. Detailed trait ascertainment, genotyping and quality control procedures of the UK Biobank are provided in supplementary figure S1 and the supplementary material.

\section{PGC GWAS data for mental health disorders}

We retrieved summary statistics from publicly available GWAS studies from the PGC: ADHD ( $\mathrm{n}_{\text {case }} /$ $\left.\mathrm{n}_{\text {control }}=19099 / 34194\right)$ [16], ANX ( $\left.\mathrm{n}_{\text {case }} / \mathrm{n}_{\text {control }}=5710 / 11600\right)$ [17], ASD ( $\left.\mathrm{n}_{\text {case }} / \mathrm{n}_{\text {control }}=6179 / 7377\right)$ [18], $\operatorname{BIP}\left(\mathrm{n}_{\text {case }} / \mathrm{n}_{\text {control }}=7481 / 9250\right) \quad[19], \quad \mathrm{ED} \quad\left(\mathrm{n}_{\text {case }} / \mathrm{n}_{\text {control }}=3495 / 10982\right) \quad[20], \quad M D D \quad\left(\mathrm{n}_{\text {case }} / \mathrm{n}_{\text {control }}=59851 /\right.$ 113154 after excluding 23andMe) [21], PTSD $\left(\mathrm{n}_{\text {case }} / \mathrm{n}_{\text {control }}=2424 / 7113\right)$ [22] and SCZ $\left(\mathrm{n}_{\text {case }} / \mathrm{n}_{\text {control }}=34241 /\right.$ 45604) [23]. Details of each of the datasets can be found in supplementary table S1. 


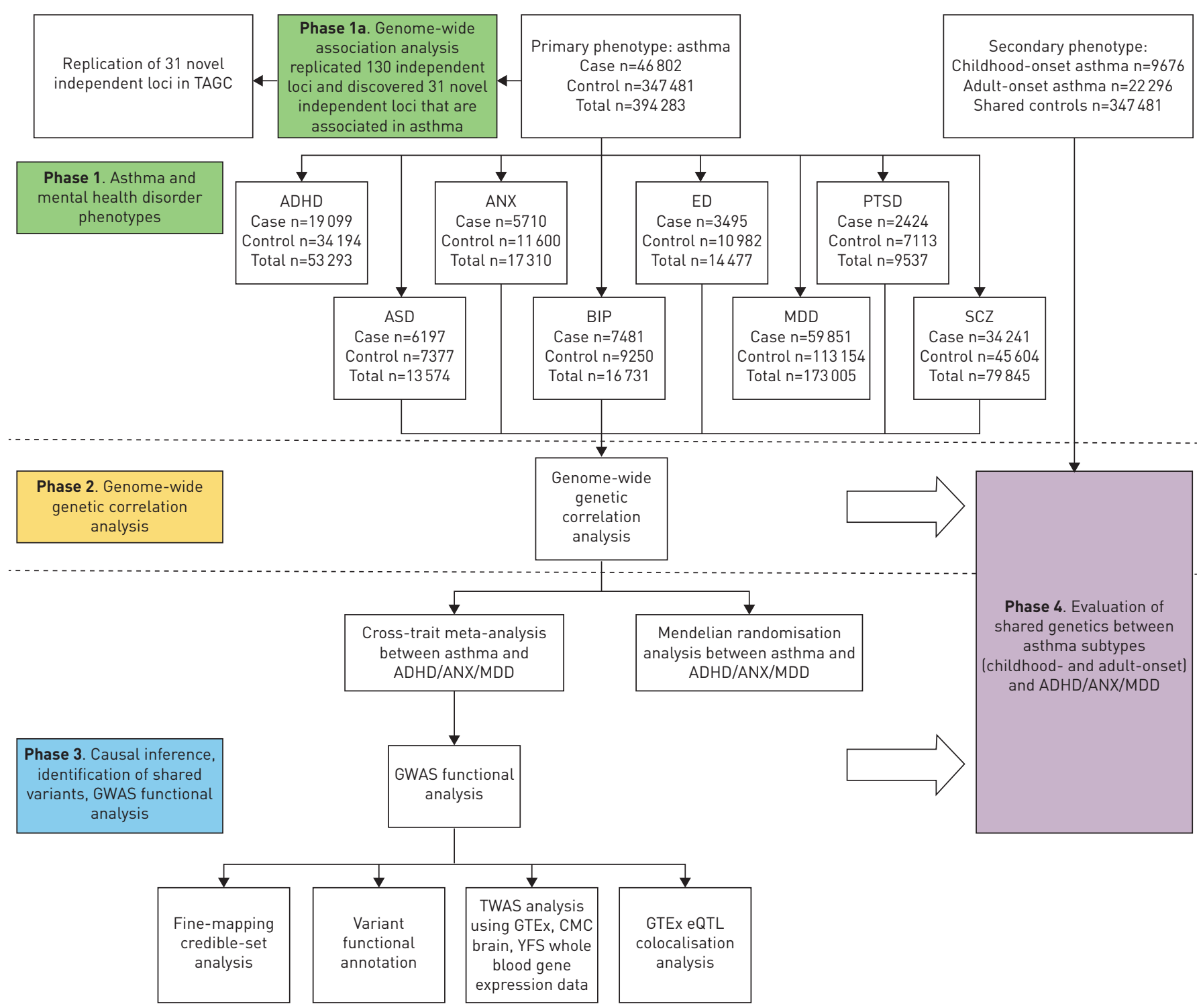

FIGURE 1 Overall study design. TAGC: Transnational Asthma Genetics Consortium; ADHD: attention deficit hyperactivity disorder; ANX: anxiety disorder; ASD: autism spectrum disorder; BIP: bipolar disorder; ED: eating disorder; MDD: major depressive disorder; PTSD: post-traumatic stress disorder; SCZ: schizophrenia; GWAS: genome-wide association study; GTEx: Genotype-Tissue Expression project; TWAS: transcriptome-wide association study; CMC: CommonMind Consortium; YFS: Young Finns Study; eQTL: expression quantitative trait loci.

We applied standardisation of GWAS summary statistics to minimise potential biases due to quality control procedures. We converted GWAS summary statistics with hg18 genome build to hg19 using the liftOver tool [24]. Indels and rare/low-frequency variants with minor allele frequency $<1 \%$ were not included in this study. Additionally, we restricted our analysis to autosomal chromosomes.

\section{GWAS analysis in the UK Biobank}

In this study, we focused on common variants for the analysis with minor allele frequency $>1 \%$. We performed stringent GWAS quality control procedures. We included variants that did not deviate from Hardy-Weinberg equilibrium $\left(\mathrm{p}>1 \times 10^{-6}\right)$, with per variant and per sample missing rates $<10 \%$, and an imputation quality score (INFO) $>0.8$. Quantile-quantile plots were produced and checked for each asthma phenotype. The linkage disequilibrium score regression (LDSC) intercept was used to evaluate genomic inflation due to population stratification. A total of 8274727 single nucleotide polymorphisms (SNPs) passed quality control on the whole genome, which were eligible for statistical association analyses.

We performed the three GWAS analyses for all asthma, childhood-onset asthma, adult-onset asthma and shared controls adjusting for age, sex, genotyping array, assess centre and 30 ancestry principal components. We did not remove any related samples in the UK Biobank since we used a linear mixed 
model (LMM) method for phenotype-genotype association analysis, which proved to be robust to potential confounding due to relatedness [25]. The output of BOLT-LMM linear regression was transformed into log odds ratio for asthma binary phenotypes. We applied the PLINK [26] clumping function (parameters: --clump-p1 5e-8 --clump-p2 1e-5 --clump-r2 0.05 --clump-kb 500) to determine top loci that are independent to each other, i.e. variants with $\mathrm{p}<1 \times 10^{-5}, \mathrm{r}^{2}>0.05$ and $<500 \mathrm{~kb}$ away from the peak will be assigned to that peak's clump. The peak variant was defined as a sentinel variant. We used the National Human Genome Research Institute-European Bioinformatics Institute (NHGRI-EBI) GWAS Catalog (www.ebi.ac.uk/gwas; search date: July 1, 2019) for checking the previously reported status of genetic loci associating with asthma and identified novel loci. Novel asthma loci were defined as the clump regions that did not contain any previously reported variants in the NHGRI-EBI GWAS Catalog.

\section{LDSC analysis}

We conducted post-GWAS genome-wide genetic correlation analysis between asthma and mental health disorders using all SNPs after merging with HapMap3 SNPs excluding the human leukocyte antigen (HLA) region. LDSC estimates genetic correlation between the true causal effects of two traits (ranging from -1 to 1) [27]. European ancestry subjects were used in LDSC analysis for each trait if available. We corrected multiple testing for LDSC p-values by the Bonferroni method and a p-value of $0.00625(0.05 / 8)$ was considered as the significance level for LDSC analysis. Mental health disorders that showed significant genome-wide genetic correlation with asthma were included in the following analyses.

\section{Cross-trait meta-analysis}

After investigating the genetic correlations among all traits, we applied association analysis based on SubSETs (ASSET; www.bioconductor.org/packages/devel/bioc/html/ASSET.html) to combine the association evidence for asthma with ADHD, ANX and MDD at individual variants since it is designed for meta-analysis of binary traits $[14,28]$. This method combines the effect estimate and standard error of the GWAS summary statistics to test the hypothesis of association between the SNP with any subset of studies.

We focused on shared sentinel variants satisfying $\mathrm{p}_{\text {meta }}<5 \times 10^{-8}$ and clump-specific false discovery rate (FDR) $<0.05$ to account for multiple testing. We used Variant Effect Predictor based on the Ensembl/ GENCODE basic transcripts database for detailed variant annotation [29].

\section{Fine-mapping credible-set analysis}

To identify the $99 \%$ credible set of variants within each of the $500 \mathrm{~kb}$ of sentinel variants, we identified a credible set of causal variants at each of the shared loci that met cross-trait meta-analysis criteria using the Bayesian-likelihood fine-mapping algorithm [30]. The Bayesian fine-mapping algorithm maps the primary signal and uses a flat prior with steepest descent approximation.

\section{Transcriptome-wide association study analysis}

To identify association of asthma with ADHD, ANX and MDD with regard to transcriptome gene expression in specific tissues, we conducted a transcriptome-wide association study (TWAS) [31] using three sets of gene expression data sources: 43 post-mortem Genotype-Tissue Expression project (GTEx) tissues $\left(n_{\text {average }}=214\right)$ [32], CommonMind Consortium $(C M C)$ brain tissue $(n=452)$ [33] and Young Finns Study (YFS) blood tissue ( $\mathrm{n}=1264$ ) [34]. Multiple testing correction for each mental health disorder was applied to account for all gene-tissue pairs based on TWAS p-values using the FDR Benjamini-Hochberg procedure (FDR $<0.05)$. Detailed statistical information can be found in the supplementary material.

\section{GTEx quantitative trait loci colocalisation analysis}

Since the GTEx expression quantitative trait loci (eQTL) signals by themselves are pervasive, we further conducted the colocalisation analysis between signals from three cross-trait meta-analysis models (asthma with ADHD, ANX and MDD) and 48 single GTEx tissues cis-eQTL (version 7) to find if the same genetic variant related to expression and the diseases. We first extracted summary association data for variants within $500 \mathrm{~kb}$ of the index SNP at each of the shared loci. We then calculated the posterior probability that the two traits (GWAS cross-trait meta-analysis and GTEx eQTL) were associated and shared one common causal variant (PPH4) [35]. Loci were considered to be colocalised with PPH4 >0.7. We conducted the tissue enrichment using permutation tests (1000 permutations) and calculated the permutation $\mathrm{p}$-values for each tissue. We considered significant enrichment based on a $\mathrm{p}$-value $=0.001042$ (0.05/48 tissues) after correcting for multiple testing of 48 tissues. 


\section{Mendelian randomisation analysis}

We applied generalised summary data-based Mendelian randomisation (GSMR) [36] under default settings to infer putative causal relationships between asthma and mental health disorders from GWAS summary statistics. GSMR requires a minimum of $10 \mathrm{LD}$-independent instruments $\left(\mathrm{r}^{2}<0.05\right)$ that are associated with exposure at the GWAS significance level $\left(\mathrm{p}<5 \times 10^{-8}\right)$ and removes SNPs displaying horizontal pleiotropy (HEIDI-outlier $\mathrm{p}<0.01$ ). Accordingly, we restricted our analyses to traits that satisfy this criterion. Additionally, we performed outlier sensitivity analysis using a more exclusive HEIDI-outlier threshold of 0.1. Prior to running GSMR, we removed strand ambiguous SNPs, poorly imputed SNPs $($ INFO $<0.9)$ and SNPs in the major histocompatibility complex region $($ chr6:25-34M).

\section{Sensitivity analysis in childhood-and adult-onset asthma}

Recent studies have shown that asthma is a highly heterogeneous disease and its genetics are partially distinct between childhood- and adult-onset asthma [12,13]. Thus, we also investigated if the shared genetics between asthma and ADHD/ANX/MDD are different with respect to childhood- and adult-onset asthma, specifically in genetic correlation, cross-trait meta-analysis, TWAS and Mendelian randomisation analyses.

\section{Results}

Phenotypic association between asthma and mental health disorders in the UK Biobank

We conducted the phenotypic association analysis using logistic regression in the UK Biobank between asthma and high-quality mental disorders based on two models: 1) unadjusted and 2) adjusted for age, sex and education. In both models, we found asthma to be significantly associated with ANX, BIP, MDD, ED and PTSD (supplementary table S2).

\section{Genome-wide association and SNP-based heritability}

There was no evidence of population stratification for three asthma GWASs (figure 2a, and supplementary figures S2 and S3). We identified 161 independent loci associated with asthma at the genome-wide significance level $\left(p<5 \times 10^{-8}\right)$, comprised of 130 previously reported loci and 31 novel loci (figure $2 b$, and supplementary tables S3 and S4). For the 31 novel loci, we conducted replication analysis in the Transnational Asthma Genetics Consortium (TAGC) data (23948 cases and 118538 controls) [37]. 21 of these loci were not found in the TAGC data, likely because the TAGC meta-analysis was based on HapMap2 imputation. Thus, we used the most significant SNP in the clump region that was available from HapMap2/TAGC as the surrogate SNP and extracted the association results from the TAGC for replication purposes. As a result, we found surrogate SNPs for 14 loci, but the remaining seven loci were not applicable for replication. Thus, a total of 24 loci were sought for replication in the TAGC. Among them, we found 14 were nominally significant in the TAGC multi-ancestry or European population $(\mathrm{p}<0.05) ; 10$ of the 14 loci had $\mathrm{p}<0.001$ (supplementary table S3). In addition, we found the effect sizes of the 24 loci were highly consistent between the UK Biobank and the TAGC (supplementary figures S4 and S5). Estimates of SNP-based heritability on the observed scale using GWAS summary statistics were (mean $\pm \mathrm{SE}$ ) $5.02 \pm 0.62 \%$ for asthma, $3.38 \pm 0.66 \%$ for childhood-onset asthma and $1.98 \pm 0.24 \%$ for adult-onset asthma.

\section{Genome-wide genetic correlation}

We investigated the genetic correlations of asthma and mental health disorders using LDSC (table 1). We observed positive genetic correlation $\left(\mathrm{R}_{\mathrm{g}}\right)$ between asthma and ADHD $\left(\mathrm{R}_{\mathrm{g}}=0.197, \mathrm{p}=1.21 \times 10^{-5}\right)$, ANX $\left(\mathrm{R}_{\mathrm{g}}=0.406, \mathrm{p}=1.61 \times 10^{-3}\right)$ and $\mathrm{MDD}\left(\mathrm{R}_{\mathrm{g}}=0.215, \mathrm{p}=1.09 \times 10^{-8}\right)$. We did not find significant genetic correlation between asthma and other mental health disorders.

\section{Cross-trait meta-analysis between asthma and mental health disorders}

We applied ASSET for genome-wide cross-trait meta-analysis to identify genetic loci associated with asthma and ADHD, ANX and MDD ( $\mathrm{p}_{\text {meta }}<5 \times 10^{-8}$, single-trait FDR $<0.05$ ). After pruning, we found seven loci significantly associated with asthma and ADHD. The most significant SNP was rs2025758 $\left(\mathrm{p}_{\text {meta }}=4.52 \times 10^{-18}, \mathrm{FDR}_{\mathrm{asthma}}=1.29 \times 10^{-14}, \mathrm{FDR}_{\mathrm{ADHD}}=1.09 \times 10^{-3}\right)$, located at an intergenic region. We also found the HLA locus (sentinel SNP: rs3117006, $\mathrm{p}_{\text {meta }}=2.81 \times 10^{-8}, \quad \mathrm{FDR}_{\mathrm{asthma}}=6.61 \times 10^{-7}$, $\mathrm{FDR}_{\mathrm{ADHD}}=3.13 \times 10^{-2}$ ) shared by asthma and ADHD. Furthermore, we found one locus significantly associated with both asthma and ANX (sentinel SNP: $\mathrm{rs} 1709393, \mathrm{p}_{\text {meta }}=4.29 \times 10^{-8}, \mathrm{FDR}_{\mathrm{asthma}}=2.30 \times 10^{-4}$, $\mathrm{FDR}_{\mathrm{ANX}}=2.06 \times 10^{-6}$ ). In addition, we identified 10 loci significantly associated with asthma and MDD. The top sentinel SNP was $\mathrm{rs} 2855812\left(\mathrm{p}_{\text {meta }}=2.1 \times 10^{-16}, \mathrm{FDR}_{\mathrm{asthma}}=7.64 \times 10^{-13}, \mathrm{FDR}_{\mathrm{MDD}}=1.07 \times 10^{-5}\right.$ ), where its clump covers many genes in the region, mainly including HLA genes. Notably, we found two regions shared by multiple traits: 5q21.2 and the HLA region shared by asthma, ADHD and MDD (table 2 and supplementary table S5). 

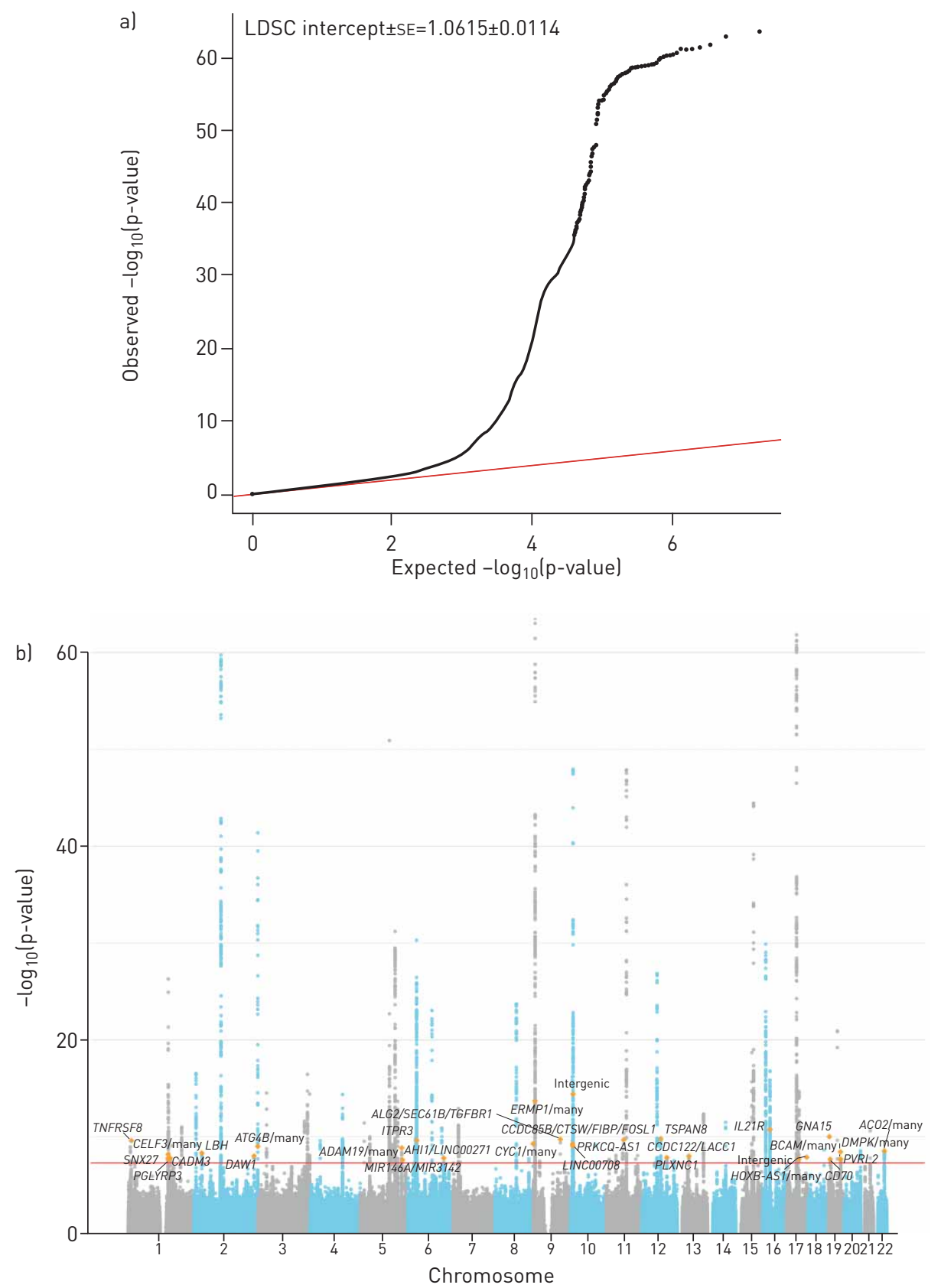

FIGURE 2 Results of genome-wide association analysis of the UK Biobank cohort for asthma. LDSC: linkage disequilibrium score regression. a) Association test quantile-quantile plot showing departure from the null hypothesis of no association. b) Manhattan plot for association test of 46802 asthma cases and 347481 controls. $\mathrm{n}=31$ novel independent loci. The most significant novel single nucleotide polymorphism in each locus is highlighted with an orange diamond. The genome-wide significance level after accounting for multiple testing $\left(p=5 \times 10^{-8}\right)$ is denoted by the red line.

\section{Identification of causal exonic missense variants}

We identified a credible set of causal SNPs using Bayesian fine-mapping at each shared locus meeting significance criteria in the asthma-mental health disorders meta-analysis. The credible set of variants at each locus was $99 \%$ likely to contain the causal variant. Lists of credible sets of SNPs for each locus are provided in supplementary tables S6-S8.

We found one locus (in BX927320.1) for asthma and MDD (supplementary table S9) in which the credible set included exonic missense polymorphisms. However, we did not find any exonic missense polymorphisms in the credible set of SNPs for asthma and ADHD or asthma and ANX (supplementary 


\begin{tabular}{|c|c|c|c|c|}
\hline Phenotype 1 & Phenotype 2 & $R_{g} \pm S E$ & $\mathbf{z}$ & $p$-value \\
\hline \multirow{8}{*}{ Asthma } & ADHD & $0.197 \pm 0.045$ & 4.376 & $1.21 \times 10^{-5}$ \\
\hline & ANX & $0.406 \pm 0.129$ & 3.155 & $1.61 \times 10^{-3}$ \\
\hline & ASD & $0.017 \pm 0.052$ & 0.324 & $7.46 \times 10^{-1}$ \\
\hline & BIP & $0.085 \pm 0.054$ & 1.560 & $1.19 \times 10^{-1}$ \\
\hline & ED & $0.003 \pm 0.055$ & 0.045 & $9.64 \times 10^{-1}$ \\
\hline & MDD & $0.215 \pm 0.038$ & 5.717 & $1.09 \times 10^{-8}$ \\
\hline & PTSD & $0.458 \pm 0.471$ & 0.972 & $3.31 \times 10^{-1}$ \\
\hline & $\mathrm{SCZ}$ & $0.012 \pm 0.026$ & 0.471 & $6.38 \times 10^{-1}$ \\
\hline
\end{tabular}

$\mathrm{R}_{\mathrm{g}}$ : genetic correlation estimate; ADHD: attention deficit hyperactivity disorder; ANX: anxiety disorder; ASD: autism spectrum disorder; BIP: bipolar disorder; ED: eating disorder; MDD: major depressive disorder; PTSD: post-traumatic stress disorder; SCZ: schizophrenia.

tables S10 and S11), since most variants were either intronic or intergenic, aligning with the theory that most variants identified by GWAS involve gene regulatory effects rather than protein structure changes [38].

\section{TWAS and GTEX eQTL colocalisation}

To investigate specific tissue-gene pairs that are shared by asthma and mental health disorders, we further performed TWAS analysis on asthma, ADHD, MDD and ANX using three gene expression data sources. We investigated the overlap of significant tissue-gene pairs in asthma and ADHD, MDD and ANX. There was an overlap in 18 significant tissue-gene pairs in GTEx and three pairs in CMC brain for asthma and ADHD. There was an overlap in one significant tissue-gene pair in YFS blood for asthma and MDD (table 3). No overlapped tissue-gene pair was found for asthma and ANX. Since CMC brain and YFS blood gene expression datasets have larger sample sizes than GTEx, for tissues of brain and blood we considered CMC and YFS as discovery datasets and GTEx as the replication dataset. We additionally extracted the association statistics of four significant tissue-gene pairs between asthma and mental health disorders (CISD2, KATNA1 and MANBA from CMC brain; POLI from YFS blood) from the GTEx results. We replicated all of them in the GTEx dataset accounting for multiple testing for the available genes ( $\mathrm{p}<0.05 /$ three genes) except for KATNA1, which is not available in GTEx brain tissues (supplementary table S12).

We further conducted colocalisation analysis for the shared genetic variants from cross-trait meta-analysis between asthma and ADHD, ANX and MDD with GTEx eQTLs across 48 tissues. For asthma and $\mathrm{ADHD}$, we found shared variants at the 10p14 region (e.g. GATA3), 4q24 (e.g. MANBA) and the HLA region were the potential causal eQTL variants in many tissues (supplementary table S13). Notably, in asthma and MDD, HLA was also the major causal eQTL colocalised region (supplementary table S14). Through the permutation analysis, we observed a significant amount of colocalised signals between asthma and $\mathrm{ADHD} / \mathrm{MDD}$ in some specific tissues, belonging mainly to the exocrine/endocrine, digestive, respiratory and haemic/immune systems (supplementary figures S6 and S7).

\section{Mendelian randomisation results}

We observed a small but significant positive causal effect of ADHD on asthma $\left(\beta_{\mathrm{ADHD} \rightarrow \mathrm{Asthma}}=0.054\right.$, $\mathrm{p}=0.036$ ), but not vice versa (table 4), corroborating the putative model that ADHD causally increases the risk of asthma. We also observed a strongly significant positive causal effect of MDD on asthma $\left(\beta_{\mathrm{MDD} \rightarrow \text { Asthma }}=0.21, \mathrm{p}=1.80 \times 10^{-5}\right)$. However, since there is a small fraction of overlapping samples between MDD and asthma GWAS data $(\sim 6.7 \%)$ and potential unobserved confounders, our Mendelian randomisation conclusion should be interpreted with caution. Due to limited power of the GWAS, we could not identify causal relationships between asthma and ANX.

\section{Sensitivity analysis in childhood- and adult-onset asthma}

We found ADHD, ANX and MDD have a positive genetic correlation with adult-onset asthma (ADHD: $\mathrm{R}_{\mathrm{g}}=0.28, \mathrm{p}=9.91 \times 10^{-6}$; ANX: $\mathrm{R}_{\mathrm{g}}=0.50, \mathrm{p}=3.29 \times 10^{-3}$; MDD: $\mathrm{R}_{\mathrm{g}}=0.34, \mathrm{p}=1.52 \times 10^{-10}$ ). We did not observe any genetic correlation between childhood-onset asthma and mental health disorders (supplementary table S15). In terms of the 18 shared genetic sentinel variants, we found some of them have stronger associations with childhood-onset asthma, but others have stronger associations with adult-onset asthma (supplementary table S16). For shared genes in the TWAS, we found most of them have approximately even associations between childhood- and adult-onset asthma, except for POLI, which has a much stronger association with childhood-onset asthma (supplementary table S17). Finally, we identified a 
TABLE 2 Genome-wide significant loci by cross-trait meta-analysis associated with asthma and attention deficit hyperactivity disorder (ADHD), anxiety disorder (ANX) or major depressive disorder (MDD) $\left(p<5 \times 10^{-8}\right.$; single-trait false discovery rate (FDR) $<0.051$

\begin{tabular}{|c|c|c|c|c|c|c|c|c|}
\hline Traits & $\begin{array}{l}\text { Sentinel } \\
\text { SNP }\end{array}$ & Genome position & A1 & A2 & FDR1 & FDR2 & $\mathrm{p}$-value & Genes within clumping region \\
\hline Asthma and ADHD & $\begin{array}{l}\text { rs } 2025758 \\
\text { rs7094182 } \\
\text { rs325485 } \\
\text { rs227283 } \\
\text { rs3117006 } \\
\text { rs6736411 } \\
\text { rs71565398 }\end{array}$ & $\begin{array}{c}\text { chr10:8 777640-8855244 } \\
\text { chr10:8 452766-8543732 } \\
\text { chr5:103769738-104048590 } \\
\text { chr4:103550006-103885568 } \\
\text { chr6:33096426-33 124972 } \\
\text { chr2:63508703-63 906628 } \\
\text { chr6:33581 633-33775446 }\end{array}$ & $\begin{array}{l}T \\
G \\
A \\
C \\
G \\
G \\
C\end{array}$ & $\begin{array}{l}C \\
C \\
G \\
G \\
A \\
A \\
G\end{array}$ & $\begin{array}{l}1.29 \times 10^{-14} \\
1.09 \times 10^{-9} \\
2.64 \times 10^{-5} \\
8.60 \times 10^{-5} \\
6.61 \times 10^{-7} \\
1.71 \times 10^{-6} \\
9.68 \times 10^{-7}\end{array}$ & $\begin{array}{l}1.09 \times 10^{-3} \\
4.89 \times 10^{-3} \\
5.31 \times 10^{-5} \\
5.58 \times 10^{-6} \\
3.13 \times 10^{-2} \\
3.45 \times 10^{-2} \\
2.18 \times 10^{-2}\end{array}$ & $\begin{array}{l}4.52 \times 10^{-18} \\
5.15 \times 10^{-12} \\
1.94 \times 10^{-8} \\
2.67 \times 10^{-8} \\
2.81 \times 10^{-8} \\
2.91 \times 10^{-8} \\
3.70 \times 10^{-8}\end{array}$ & $\begin{array}{c}\text { Intergenic region } \\
\text { Intergenic region } \\
\text { Intergenic region } \\
\text { CISD2, MANBA, SLC9B1, UBE2D3 } \\
H L A-D P B 2 \\
\text { MDH1, WDPCP } \\
\text { IP6K3, ITPR3, LEMD2, MLN, UQCC2 }\end{array}$ \\
\hline Asthma and ANX & rs1709393 & chr3:101684480-101713472 & C & $\mathrm{T}$ & $2.30 \times 10^{-4}$ & $2.06 \times 10^{-6}$ & $4.29 \times 10^{-8}$ & LOC152225 \\
\hline \multirow{9}{*}{ Asthma and MDD } & rs2855812 & chr6:31 078809-31835164 & G & $\mathrm{T}$ & $7.64 \times 10^{-13}$ & $1.07 \times 10^{-5}$ & $2.09 \times 10^{-16}$ & $\begin{array}{c}\text { ABHD16A, AIF1, APOM, ATP6V1G2, } \\
\text { ATP6V1G2-DDX39B, BAG6, C6orf15, C6orf25, } \\
\text { C6orf47, C6orf48, CCHCR1, CDSN, CLIC1, } \\
\text { CSNK2B, DDAH2, DDX39B, GPANK1, HCG26, } \\
\text { HCG27, HCP5, HLA-B, HLA-C, HSPA1A, } \\
\text { HSPA1B, HSPA1L, LSM2, LST1, LTA, LTB, } \\
\text { LY6G5B, LY6G5C, LY6G6C, LY6G6D, LY6G6E, } \\
\text { LY6G6F, MCCD1, MICA, MICB, MIR4646, } \\
\text { MIR6832, MIR6891, MSH5, MSH5-SAPCD1, } \\
\text { NCR3, NEU1, NFKBIL1, POU5F1, PRRC2A, } \\
\text { PSORS1C1, PSORS1C2, PSORS1C3, SAPCD1, } \\
\text { SLC44A4, SNORA38, SNORD48, SNORD52, } \\
\text { SNORD84, SNORD117, TCF19, TNF, VARS, } \\
\text { VWA7 }\end{array}$ \\
\hline & rs2854275 & chr6:32606970-32808299 & C & A & $1.18 \times 10^{-13}$ & $1.31 \times 10^{-2}$ & $3.47 \times 10^{-13}$ & $\begin{array}{c}H L A-D O B, H L A-D Q A 1, H L A-D Q A 2, H L A-D Q B 1 \\
H L A-D Q B 2, T A P 2\end{array}$ \\
\hline & rs396755 & chr5:103791 044-104088117 & C & G & $3.80 \times 10^{-5}$ & $9.59 \times 10^{-8}$ & $1.55 \times 10^{-11}$ & Intergenic region \\
\hline & rs150814685 & chr6:28207991-29196418 & T & G & $9.84 \times 10^{-9}$ & $1.78 \times 10^{-4}$ & $1.91 \times 10^{-11}$ & $\begin{array}{c}\text { C6orf100, GPX5, GPX6, HCG14, LOC401242, } \\
\text { LOC100129636, NKAPL, OR2B3, OR2J2, OR2J3, } \\
\text { OR2W1, PGBD1, TRIM27, ZBED9, ZKSCAN3, } \\
\text { ZKSCAN4, ZNF311, ZSCAN12, ZSCAN23, } \\
\text { ZSCAN26, ZSCAN31 }\end{array}$ \\
\hline & rs149702363 & chr6:29 218513-29 448128 & $\mathrm{~T}$ & G & $1.33 \times 10^{-8}$ & $2.50 \times 10^{-3}$ & $3.55 \times 10^{-1}$ & $\begin{array}{c}\text { OR2H1, OR5V1, OR10C1, OR11A1, OR12D2, } \\
\text { OR12D3, OR14J1 }\end{array}$ \\
\hline & rs301817 & $\operatorname{chr} 1: 8415235-8847380$ & C & $A$ & $1.59 \times 10^{-9}$ & $4.59 \times 10^{-2}$ & $2.94 \times 10^{-9}$ & RERE \\
\hline & rs148696809 & chr6:28934352-28934352 & T & C & $1.53 \times 10^{-3}$ & $1.96 \times 10^{-7}$ & $4.78 \times 10^{-9}$ & Intergenic region \\
\hline & rs147121091 & chr6:27 174557-27 765899 & A & G & $1.87 \times 10^{-4}$ & $4.58 \times 10^{-5}$ & $2.63 \times 10^{-8}$ & $\begin{array}{c}\text { LINC01012, LOC100131289, POM121L2, } \\
\text { PRSS16, VN1R10P, ZNF184, ZNF204P, ZNF391 }\end{array}$ \\
\hline & rs10789340 & chr1:72565800-72945128 & A & G & $1.44 \times 10^{-3}$ & $3.36 \times 10^{-6}$ & $2.68 \times 10^{-8}$ & NEGR1 \\
\hline
\end{tabular}

SNP: single nucleotide polymorphism; A1: effect allele; A2: reference allele; FDR: false discovery rate.

modest but nonsignificant causal effect between ADHD and childhood/adult-onset asthma. We also observed a strong positive causal effect of MDD on adult-onset asthma $\left(\beta_{\mathrm{MDD} \rightarrow \text { Adult-onset asthma }}=0.26\right.$, $\left.\mathrm{p}=3.00 \times 10^{-4}\right)$ (supplementary table S18).

\section{Discussion}

To the best of our knowledge, this study is the largest genome-wide analysis that has investigated the genetic overlap between asthma and mental health disorders. In this study, we identified 161 independent loci associated with asthma at a genome-wide significance level, which contains 130 previously reported loci and 31 novel loci. More than half of the novel loci were replicated in the TAGC cohort. We also 
TABLE 3 Significant overlap transcriptome-wide association analysis results between asthma and attention deficit hyperactivity disorder (ADHD) or major depressive disorder (MDD) (false discovery rate (FDR) <0.05)

\begin{tabular}{|c|c|c|c|c|c|c|c|c|}
\hline \multirow[t]{2}{*}{ Tissue } & \multirow[t]{2}{*}{ Gene } & \multirow[t]{2}{*}{ Chromosome } & \multirow[t]{2}{*}{ SNPs $\mathrm{n}^{\#}$} & \multicolumn{2}{|c|}{ Asthma } & \multicolumn{3}{|c|}{ Mental health disorder } \\
\hline & & & & Best.GWAS.ID & FDR & Best.GWAS.ID & FDR & Trait \\
\hline \multicolumn{9}{|l|}{ GTEx } \\
\hline Artery_Aorta & CISD2 & 4 & 326 & rs227375 & 0.038 & rs227369 & 0.035 & ADHD \\
\hline Cells_Transformed_fibroblasts & MANBA & 4 & 389 & rs227375 & 0.009 & rs227369 & 0.001 & ADHD \\
\hline Cells_Transformed_fibroblasts & UBE2D3 & 4 & 343 & rs227375 & 0.045 & rs227369 & 0.036 & ADHD \\
\hline Esophagus_Gastroesophageal_Junction & CISD2 & 4 & 326 & rs227375 & 0.049 & rs227369 & 0.036 & ADHD \\
\hline Esophagus_Muscularis & CISD2 & 4 & 326 & rs227375 & 0.022 & rs227369 & 0.025 & ADHD \\
\hline Lung & CISD2 & 4 & 326 & rs227375 & 0.038 & rs227369 & 0.025 & ADHD \\
\hline Muscle_Skeletal & CISD2 & 4 & 326 & rs227375 & 0.004 & rs227369 & 0.004 & ADHD \\
\hline Ovary & CISD2 & 4 & 326 & rs227375 & 0.032 & rs227369 & 0.032 & ADHD \\
\hline Pancreas & MANBA & 4 & 389 & rs227375 & 0.013 & rs227369 & 0.039 & ADHD \\
\hline Spleen & MANBA & 4 & 389 & rs227375 & 0.038 & rs227369 & 0.005 & ADHD \\
\hline Uterus & CISD2 & 4 & 326 & rs227375 & 0.038 & rs227369 & 0.007 & ADHD \\
\hline \multicolumn{9}{|l|}{ CMC } \\
\hline Brain & CISD2 & 4 & 322 & rs227375 & 0.003 & rs227369 & 0.010 & ADHD \\
\hline Brain & KATNA1 & 6 & 469 & rs112225 & 0.010 & rs2342764 & 0.048 & ADHD \\
\hline Brain & MANBA & 4 & 390 & rs228617 & 0.009 & rs227369 & 0.009 & ADHD \\
\hline \multicolumn{9}{|l|}{ YFS } \\
\hline Blood & POLI & 18 & 347 & rs3730783 & 0.016 & rs4801003 & 0.046 & MDD \\
\hline
\end{tabular}

SNP: single nucleotide polymorphism; GWAS: genome-wide association study; ID: identifier; CMC: CommonMind Consortium; YFS: Young Finns Study. " : number of SNPs in the locus.

showed a strong positive genetic correlation between asthma and three mental health disorders (ADHD, ANX and MDD).

We also identified the genetic overlap between asthma and ADHD, ANX or MDD at the individual variant level, including seven loci shared by asthma and ADHD, one loci shared by asthma and ANX, and 10 loci shared by asthma and MDD from cross-trait meta-analysis. We highlighted the HLA region (several sentinel SNPs) for its significant role in between asthma and mental health disorders. The HLA region harbours more than 200 genes located close to each other on chromosome 6 . It is a gene complex that contains abundant pleiotropy for many complex diseases, especially those involved in immune-related

TABLE 4 Estimates of causal effect size between asthma and mental health disorders

\begin{tabular}{lccccc} 
Phenotype 1 & Phenotype 2 & Direction & Causal effect size \pm SE & p-value & Instruments n \\
\hline ADHD & Asthma & $\rightarrow$ & $\begin{array}{c}0.054 \pm 0.026 \\
-0.034 \pm 0.025\end{array}$ & $\begin{array}{c}0.036 \\
0.16\end{array}$ & 10 \\
& & $\leftarrow$ & NA & NA & NA \\
\hline \multirow{2}{*}{ ANX } & Asthma & $\rightarrow^{\#}$ & $-0.014 \pm 0.055$ & 0.8 & 159 \\
& & $\leftarrow$ & $0.21 \pm 0.049$ & $1.80 \times 10^{-5}$ & 20 \\
\multirow{2}{*}{ MDD } & Asthma & $\rightarrow^{\Uparrow}$ & $0.012 \pm 0.015$ & 0.45 & 124 \\
& & $\leftarrow$ &
\end{tabular}

" $\rightarrow$ " refers to the phenotype $1 \rightarrow$ phenotype 2 causal direction; " $\leftarrow$ " refers to the phenotype $2 \rightarrow$ phenotype 1 causal direction. ADHD: attention deficit hyperactivity disorder; ANX: anxiety disorder; MDD: major depressive disorder; NA: not available; GWAS: genome-wide association study. ${ }^{\#}$ : the ANX GWAS does not have enough single nucleotide polymorphisms at the genome-wide significance level for constructing the instrument variable; " : the MDD GWAS data include 23 andMe. 
process [39]. WANG et al. [40] also identified that the HLA region showed the strongest role contributing to the pleiotropic effect between psychiatric and immune disorders, although asthma was not assessed in their study. For example, with the inclusion of the HLA region, the pleiotropy significance between SCZ and rheumatoid arthritis was around 280 times stronger than with the exclusion of the HLA region [40].

Furthermore, we investigated whether genes shared between asthma and mental health disorders have a potential functional connection with human tissues. In the TWAS analysis, we found multiple shared tissue-gene pairs between asthma and $\mathrm{ADHD}$, including the exocrine/endocrine, digestive, respiratory and nervous systems. Of them, CISD2 was found to be shared between asthma and ADHD in most of the tissues and potentially have a significant biological function. CDGSH iron sulfur domain 2 (CISD2) deficiency causes mitochondrial breakdown and dysfunction, and drives premature ageing [41]. A transmission electron microscopy study revealed that mitochondrial degeneration occurs in brain cells and skeletal muscle cells in Cisd2 $2^{-/}$mice [41]. Mitochondrial dysfunction was associated with allergic asthma [42] and affected the digestive system. Mitochondrial defects are also detected in ADHD cybrids created from patients' platelets, implying mitochondrial dysfunction could be a contributory factor for ADHD pathology [43]. In addition, we found the POLI gene in YFS whole blood tissue was shared by asthma and MDD. One possible mechanism for such a connection is through DNA polymerase $\eta$, which arises from a gene duplication from DNA polymerase $i$ encoded by the POLI gene. DNA polymerase $\eta$ is the sole contributor of A/T modifications during immunoglobulin gene hypermutation in the mouse [44]. IgE is a key component in the pathology of asthma. Recognition of allergens by IgE depends on a combination of choice of human immunoglobulin heavy chain variable genes, utilisation of certain mutational hotspots and improvement of affinity via additional mutations in complementarity determining regions [45]. DNA polymerase $\eta$ also modulates DNA damage response and DNA damage is the key to treat many of the genetically inherited central nervous system disorders including depression [46].

In this study, we also investigated causal relationships between asthma and mental health disorders using Mendelian randomisation. Our results suggested that ADHD and MDD might increase the risk of asthma, providing insights into the pathological mechanisms of asthma. Due to limited power, we were not able to perform bidirectional Mendelian randomisation for ANX and asthma. In addition, our Mendelian randomisation analyses using a more exclusive outlier p-value threshold of 0.1 showed most of the Mendelian randomisation analysis results remain unchanged (supplementary table S19). We emphasise that our inferred causal relationships are putative as all Mendelian randomisation analyses in this study are based on GWAS summary statistics; unobserved confounders and overlapping samples may lead to false conclusions. Further analysis, such as gene function biological experiments and longitudinal studies, would confirm the inferred causal relationships.

In the asthma subtype sensitivity analysis, we found the shared genetics between asthma and mental health disorders are distinct for childhood- and adult-onset asthma. In terms of the genome-wide genetic correlation, childhood-onset asthma did not show a genetic correlation with any of the mental health disorders, whereas several studies observed robust phenotypic correlations between asthma and ADHD in children and adults $[3,47]$. Such findings suggest the phenotypic correlation between asthma and ADHD in children maybe more attributed to environmental factors, but not substantially from genetic origins [47]. Furthermore, our genetic correlation results suggest the genetic predisposition on ADHD (the majority are children) might have more impact on the genetic predisposition of adult-onset asthma. However, there is limited research demonstrating the phenotypic correlation between childhood-onset ADHD and adult-onset asthma, which could be investigated by longitudinal studies in the future. However, for the top genes identified in cross-trait meta-analysis and TWAS, we found the genetic effects are similar in both childhood- and adult-onset asthma, which suggests the shared genetics among complex diseases may be different at the genome-wide polygenic level and top association level. Also, LEHTO et al. [5] recently reported on the shared genetics between asthma and depression and high neuroticism in adults, based on their analysis of genome-wide genetic correlation and polygenic risk score. Complementary with the LEHTO et al. [5] study, we further examined the shared genetics at the variant, gene and tissue function levels for both childhood- and adult-onset asthma, and we fully utilised the genetic effect to infer the potential genetic causality of the observed associations.

We acknowledge several potential limitations in this study. First, the statistical power of our GWAS analysis was restricted to the sample sizes of each of the mental health disorders; genetic correlation between asthma and additional mental health disorders may be discovered with larger sample sizes. Second, the asthma information in the UK Biobank is about lifetime asthma diagnosis without information about current asthma or asthma duration. Thus, we were not able to align occurrence of asthma and mental health disorders. Third, in the asthma subtype analysis, it would be ideal to find corresponding well-powered childhood- and adult-onset mental health disorders for matched analysis with childhood- and adult-onset asthma. However, such mental health disorder GWAS data are currently 
unavailable. In addition, other asthma endotypes, such as by IgE (allergic status) and eosinophil level (type 2 inflammation) [48], may provide additional insights into the pathophysiological connection between asthma and mental health disorders. Finally, it is important to evaluate the common nongenetic risks for morbidity and mortality in asthma and mental health disorders, such as environmental and social factors. For example, inhaled corticosteroid, the most common medication for asthma, which is not available in the UK Biobank data, may have potential adverse effects on mental health, such as depression and anxiety [49]. The current study was limited to assessing shared genetic factors between asthma and mental health disorders, and future studies on shared environmental factors between them are needed.

\section{Conclusions}

Understanding the genetic overlap between asthma and mental health disorders may be beneficial to the management of both conditions. Our study shows evidence of significant positive genetic correlations between asthma and three mental health disorders. Shared genetic variants were fine-mapped to improve resolution and identify potential shared causal variants with exonic missense polymorphisms. We also found multiple potential common biological mechanisms, which can advance our understanding of the connection between asthma and some mental health disorders, and offer new avenues for future functional validation, disease prevention and clinical treatment.

Acknowledgements: This research was conducted using the UK Biobank Resource under application numbers 16549 and 45052. We would like to thank participants and researchers from the UK Biobank who significantly contributed or collected data. We are grateful to all participants from the Psychiatric Genomics Consortium for their significant contributions to share data.

Author contributions: Z. Zhu, X. Zhu, K. Hasegawa, C.A. Camargo and L. Liang designed the study. Z. Zhu and H. Shi performed the statistical analysis. Z. Zhu, X. Zhu, C-L. Liu, S. Shen, Y. Yang, K. Hasegawa and C.A. Camargo wrote the first draft of the manuscript. All authors helped interpret the data, reviewed and edited the final paper, and approved the submission. Z. Zhu and L. Liang had full access to all the data in the study, and take responsibility for the integrity of the data and the accuracy of the data analysis.

Conflict of interest: None declared.

\section{References}

1 Wright RJ, Rodriguez M, Cohen S. Review of psychosocial stress and asthma: an integrated biopsychosocial approach. Thorax 1998; 53: 1066-1074.

2 Van Lieshout RJ, Macqueen G. Psychological factors in asthma. Allergy Asthma Clin Immunol 2008; 4: 12-28.

3 Cortese S, Sun S, Zhang J, et al. Association between attention deficit hyperactivity disorder and asthma: a systematic review and meta-analysis and a Swedish population-based study. Lancet Psychiatry 2018; 5: 717-726.

$4 \quad$ Wamboldt MZ, Hewitt JK, Schmitz S, et al. Familial association between allergic disorders and depression in adult Finnish twins. Am J Med Genet 2000; 96: 146-153.

5 Lehto K, Pedersen NL, Almqvist C, et al. Asthma and affective traits in adults: a genetically informative study. Eur Respir J 2019; 53: 1802142.

6 Zhu Z, Lee PH, Chaffin MD, et al. A genome-wide cross-trait analysis from UK Biobank highlights the shared genetic architecture of asthma and allergic diseases. Nat Genet 2018; 50: 857-864.

7 Lee $\mathrm{PH}$, Anttila V, Won $\mathrm{H}$, et al. Genome wide meta-analysis identifies genomic relationships, novel loci, and pleiotropic mechanisms across eight psychiatric disorders [preprint]. BioRxiv 2019: 528117.

8 Zhu Z, Lin Y, Li X, et al. Shared genetic architecture between metabolic traits and Alzheimer's disease: a large-scale genome-wide cross-trait analysis. Hum Genet 2019; 138: 271-285.

9 Zhu Z, Wang X, Li X, et al. Genetic overlap of chronic obstructive pulmonary disease and cardiovascular disease-related traits: a large-scale genome-wide cross-trait analysis. Respir Res 2019; 20: 64.

10 Torgerson DG, Ampleford EJ, Chiu GY, et al. Meta-analysis of genome-wide association studies of asthma in ethnically diverse North American populations. Nat Genet 2011; 43: 887-892.

11 Bouchard J Jr. Genetic influence on human psychological traits: a survey. Curr Dir Psychol Sci 2004; 13: 148-151.

12 Ferreira MAR, Mathur R, Vonk JM, et al. Genetic architectures of childhood- and adult-onset asthma are partly distinct. Am J Hum Genet 2019; 104: 665-684.

13 Pividori M, Schoettler N, Nicolae DL, et al. Shared and distinct genetic risk factors for childhood-onset and adult-onset asthma: genome-wide and transcriptome-wide studies. Lancet Respir Med 2019; 7: 509-522.

14 Zhu Z, Anttila V, Smoller JW, et al. Statistical power and utility of meta-analysis methods for cross-phenotype genome-wide association studies. PLoS One 2018; 13: e0193256.

15 Sudlow C, Gallacher J, Allen N, et al. UK Biobank: an open access resource for identifying the causes of a wide range of complex diseases of middle and old age. PLoS Med 2015; 12: e1001779.

16 Demontis D, Walters RK, Martin J, et al. Discovery of the first genome-wide significant risk loci for attention deficit/hyperactivity disorder. Nat Genet 2019; 51: 63-75.

17 Otowa T, Hek K, Lee M, et al. Meta-analysis of genome-wide association studies of anxiety disorders. Mol Psychiatry 2016; 21: 1391-1399.

18 Autism Spectrum Disorders Working Group of the Psychiatric Genomics Consortium. Meta-analysis of GWAS of over 16,000 individuals with autism spectrum disorder highlights a novel locus at 10q24.32 and a significant overlap with schizophrenia. Mol Autism 2017; 8: 21.

19 Psychiatric GWAS Consortium Bipolar Disorder Working Group. Large-scale genome-wide association analysis of bipolar disorder identifies a new susceptibility locus near ODZ4. Nat Genet 2011; 43: 977-983. 
Duncan L, Yilmaz Z, Gaspar H, et al. Significant locus and metabolic genetic
genome-wide association study of anorexia nervosa. Am J Psychiatry 2017; 174: 850-858. Wray NR, Ripke S, Mattheisen M, et al. Genome-wide association analyses identify 44 risk variants and refine the genetic architecture of major depression. Nat Genet 2018; 50: 668-681.

22 Duncan LE, Ratanatharathorn A, Aiello AE, et al. Largest GWAS of PTSD $(N=20070)$ yields genetic overlap with schizophrenia and sex differences in heritability. Mol Psychiatry 2018; 23: 666-673.

23 Schizophrenia Working Group of the Psychiatric Genomics Consortium. Biological insights from 108 schizophrenia-associated genetic loci. Nature 2014; 511: 421-427.

25 Loh P-R, Tucker G, Bulik-Sullivan BK, et al. Efficient Bayesian mixed-model analysis increases association power in large cohorts. Nat Genet 2015; 47: 284-290.

26 Purcell S, Neale B, Todd-Brown K, et al. PLINK: a tool set for whole-genome association and population-based linkage analyses. Am J Hum Genet 2007; 81: 559-575.

27 Bulik-Sullivan B, Finucane HK, Anttila V, et al. An atlas of genetic correlations across human diseases and traits. Nat Genet 2015; 47: 1236-1241.

28 Bhattacharjee S, Rajaraman $\mathrm{P}$, Jacobs $\mathrm{KB}$, et al. A subset-based approach improves power and interpretation for the combined analysis of genetic association studies of heterogeneous traits. Am J Hum Genet 2012; 90: 821-835.

29 Zerbino DR, Achuthan P, Akanni W, et al. Ensembl 2018. Nucleic Acids Res 2018; 46: D754-D761.

30 Huang G, Liu Z, Van Der Maaten L, et al. Densely connected convolutional networks [preprint]. arXiv 2018: 1608.06993v5.

31 Gusev A, Ko A, Shi $\mathrm{H}$, et al. Integrative approaches for large-scale transcriptome-wide association studies. Nat Genet 2016; 48: 245-252.

32 GTEx Consortium. The Genotype-Tissue Expression (GTEx) project. Nat Genet 2013; 45: 580-585.

33 Fromer M, Roussos P, Sieberts SK, et al. Gene expression elucidates functional impact of polygenic risk for schizophrenia. Nat Neurosci 2016; 19: 1442-1453.

34 Raitakari OT, Juonala M, Ronnemaa T, et al. Cohort profile: the cardiovascular risk in Young Finns Study. Int J Epidemiol 2008; 37: 1220-1226.

35 Wallace C. Statistical testing of shared genetic control for potentially related traits. Genet Epidemiol 2013; 37: 802-813.

36 Zhu Z, Zheng Z, Zhang F, et al. Causal associations between risk factors and common diseases inferred from GWAS summary data. Nat Commun 2018; 9: 224.

38 Maurano MT, Humbert R, Rynes E, et al. Systematic localization of common disease-associated variation in regulatory DNA. Science 2012; 337: 1190-1195.

39 Sivakumaran S, Agakov F, Theodoratou E, et al. Abundant pleiotropy in human complex diseases and traits. Am J Hum Genet 2011; 89: 607-618.

40 Wang Q, Yang C, Gelernter J, et al. Pervasive pleiotropy between psychiatric disorders and immune disorders revealed by integrative analysis of multiple GWAS. Hum Genet 2015; 134: 1195-1209.

41 Chen YF, Kao CH, Chen YT, et al. Cisd2 deficiency drives premature aging and causes mitochondria-mediated defects in mice. Genes Dev 2009; 23: 1183-1194.

42 Mabalirajan U, Dinda AK, Kumar S, et al. Mitochondrial structural changes and dysfunction are associated with experimental allergic asthma. J Immunol 2008; 181: 3540-3548.

43 Verma P, Singh A, Nthenge-Ngumbau DN, et al. Attention deficit-hyperactivity disorder suffers from mitochondrial dysfunction. BBA Clin 2016; 6: 153-158.

44 Delbos F, Aoufouchi S, Faili A, et al. DNA polymerase eta is the sole contributor of A/T modifications during immunoglobulin gene hypermutation in the mouse. J Exp Med 2007; 204: 17-23.

45 Snow RE, Chapman CJ, Frew AJ, et al. Pattern of usage and somatic hypermutation in the $\mathrm{V}_{\mathrm{H}} 5$ gene segments of a patient with asthma: implications for IgE. Eur J Immunol 1997; 27: 162-170.

46 Black CN, Bot M, Scheffer PG, et al. Is depression associated with increased oxidative stress? A systematic review and meta-analysis. Psychoneuroendocrinology 2015; 51: 164-175.

47 Biederman J, Milberger S, Faraone SV, et al. Associations between childhood asthma and ADHD: issues of psychiatric comorbidity and familiality. J Am Acad Child Adolesc Psychiatry 1994; 33: 842-848.

48 Froidure A, Mouthuy J, Durham SR, et al. Asthma phenotypes and IgE responses. Eur Respir J 2016; 47: $304-319$.

49 Hyun MK, Lee NR, Jang EJ, et al. Effect of inhaled drugs on anxiety and depression in patients with chronic obstructive pulmonary disease: a prospective observational study. Int J Chron Obstruct Pulmon Dis 2016; 11: $747-754$ 